\title{
Changes in the calling behaviour of female Spodoptera littoralis (Lepidoptera: Noctuidae) as a function of body weight and adult feeding
}

\author{
Medhat M. SADEK \\ Department of Zoology, Faculty of Science, Assiut University, Assiut 71516, Egypt; e-mail: m.sadek@aun.edu.eg
}

Key words. Lepidoptera, Noctuidae, Spodoptera littoralis, body weight, calling behaviour, adult food

\begin{abstract}
Because nutrients accrued during larval stages represent the major limiting factor for egg production, the use of adult feeding to enhance the reproductive output in moths is considered to be largely weight-dependent. It is hypothesized, however, that feeding by adults could be adaptive and an effective means of increasing their reproductive success. In order to test this, the calling behaviour of Spodoptera littoralis females that differed in body weight and whether they had fed or not were recorded. Two experiments were carried out. In the first, the calling behaviour of food-deprived females of different body weights was recorded. A strong positive correlation was found between body weight at emergence and the total duration of calling of females on the second to the fifth night after emergence. In the second experiment, groups of female moths that varied in body weight were given access to water or sucrose. Feeding on sucrose significantly reduced the pre-calling period and increased the total time spent calling on the six nights after emergence. The increase in time spent calling associated with ingesting sucrose were proportionately similar for both small and large females, implying that feeding by adults can result in an increase in the time spent calling by moths irrespective of larval nutritional status. Female longevity was also correlated with moth weight at emergence and/or sucrose availability. It is concluded that it is advantageous for female $S$. littoralis to be large and/or have access to sucrose-rich food in the adult stage as they can spend more time attracting a mate, which increases their chances of mating in early adult life, and their longer adult life may indirectly result in an increase in fecundity.
\end{abstract}

\section{INTRODUCTION}

Body weight is one of the most important factors shaping biological processes in insects (Reavey, 1993). Large female insects are generally thought to be potentially very fecund (Honek, 1993; Tammaru et al., 2002; Xu \& Wang, 2009). In addition, various aspects of the reproductive physiology and behaviour of moths, e.g. sexual selection (Jaffe et al., 2007; Xu \& Wang, 2009; Harari et al., 2011), propensity for re-mating (Torres-Vila et al., 1997) and investment by males (Teng \& Zhang, 2009) are also affected by body weight. Calling behaviour, in which females release sex pheromones to attract males, is a prerequisite for mating in moths. Many studies have shown that calling is affected by endogenous and exogenous factors, including temperature (Mozuraitis \& Buda, 2006), photoperiod (Gomez \& Rojas, 2006), age (Ambrogi et al., 2009), mating status (McNamara et al., 2009), presence of host or non-host plant leaves (Sadek \& Anderson, 2007) and perception of the pheromone produced by conspecifics (Lim et al., 2007). Nevertheless, the effect of body weight on calling behaviour has rarely been addressed.

Variation in adult body weight in insects is an outcome of several factors, among which the availability and quality of larval food are especially important (Thiery \& Moreau, 2005; Torres-Vila et al., 2005; Liu et al., 2004). In some cases insects can compensate for poor nutrition during larval life by feeding as adults (Ryne et al., 2004). Therefore, the relation between feeding by adults and reproduction, mainly fecundity, has been frequently investigated, especially in moths where adult feeding is not necessarily required for oviposition but usually increases fecundity (Wheeler, 1996). However, these studies report mixed results regarding the role of feeding by adults and its relation to larval nutrition or adult body weight. For instance, O'Brien et al. (2002) report that although female Lepidoptera can use nectar to increase fecundity, nutrients accrued during the larval stages determine the upper limit on the use of adult dietary resources. This implies that large females, i.e. females that are well-fed as larvae, are more able to utilize food than small females. On the other hand, Gunn \& Gatehouse (1985) found that the fecundity of females originating from poorly-fed larvae, which are fed sucrose, is increased whereas that of those originating from larvae fed ad libitum but not fed as adults achieved maximum reproductive output. Fecundity has also been shown to be correlated with the weight of the moths at emergence when females are fed with water or a honey solution, but not when deprived of water or food ( $\mathrm{Gu} \&$ Danthanarayana, 1990), indicating that the effect of honey does not differ from the effect of water and both effects are weightdependent. In addition to providing energy for mating, development of eggs and oviposition (O'Brien et al., 2000), another benefit of feeding during the adult stage could be an enhancement of the likelihood of mating resulting from females increasing their calling activity. Foster (2009) and Foster \& Johnson (2010) have shown that virgin and mated females that are fed sugar produce more sex pheromone, indicating that feeding during the adult stage affects calling behaviour. However, it is 
unknown whether the putative effect of feeding during the adult stage on calling behaviour is weight-dependent.

The Egyptian cotton leaf worm, Spodoptera littoralis (Boisduval) (Lepidoptera: Noctuidae), is a serious polyphagous pest in a number of temperate and Mediterranean countries (Brown \& Dewhurst, 1975). Considerable variation in body weight of adult moths in the field is to be expected as the larvae feed on different host plants or experience different abiotic factors. Even when $S$. littoralis feeds on the same host plant under controlled conditions it can vary greatly in body weight (Sadek, 2011). Availability of nectar as a natural food for adult $S$. littoralis (Salama \& Shoukry, 1972) is always changing due to continuous changes in the vegetation profile. Information on the interaction between body weight and adult food on the reproductive behaviour of this species is of both ecological and applied importance. I hypothesize that feeding on sucrose in the adult stage increases the fitness of the moths independently of their body weight, even if it does not directly translate into increased fecundity. The aim of this study was, therefore, to examine the effects of body weight and feeding during the adult stage on the calling behaviour of $S$. littoralis.

\section{MATERIAL AND METHODS}

\section{Conditions under which the insects were reared and the adult calling behaviour was recorded}

Insects were taken from a laboratory culture reared on leaves of castor bean, Ricinus communis L., in an incubator at $25 \pm$ $0.1^{\circ} \mathrm{C}$, a $16 \mathrm{~L}: 8 \mathrm{D}$ photoperiod and $>70 \%$ relative humidity. After pupation, the pupae were sexed and the females were kept separate from males in an incubator under the same conditions until the time of the experiment. The calling behaviour was recorded in a dimly lit room (about 2 lx) as described in Sadek $\&$ Anderson (2007). The difference in temperature between this room and that in the incubator was not more than $1{ }^{\circ} \mathrm{C}$ and the relative humidity in the room was also higher than $70 \%$.

\section{Bioassays}

Two experiments were carried out. In the first experiment the relation between body weight and total time spent calling was recorded for starved females, which emerged in the same scotophase. To achieve this, females that emerged before the emergence peak were removed daily from the culture and discarded. Just prior to the scotophase (night) when most of the females were expected to emerge, pupae were transferred from the culture to the dimly lit room and the females that emerged were weighed within $10 \mathrm{~min}$ of fully expanding their wings. Each female was then placed singly in a labelled transparent plastic cylinder (length: $10 \mathrm{~cm}$, diameter: $6 \mathrm{~cm}$ ) closed with nylon fabric at both ends. The calling behaviour of 12 females was recorded on 6 successive nights starting from the first night (night 1) after emergence (night 0 ). One hour before the beginning of each night, the insects were transferred from the incubator to the dimly lit room and allowed to settle. At the beginning of the night, the dim light was turned on and calling was recorded until the end of the night, after which the females were returned to the incubator. During the recording, the tubes were mounted horizontally parallel to each other on a plastic stand and a gentle flow of air (speed $\approx 0.4 \mathrm{~m} / \mathrm{s}$ ) was pumped through the tubes to exhaust the pheromone emitted by a calling female without affecting neighboring females (Lim et al., 2007). Calling was recorded visually, as it involves a slight lifting of the wings accompanied by full protrusion of pheromone glands (Sadek \& Anderson, 2007). The moths were observed as frequently as possible and always at least once every $20 \mathrm{~min}$. The time of onset and end of calling for each female was noted. Because breaks between calling bouts in S. littoralis are usually longer than 20 min (Sadek \& Anderson, 2007), observing the insects every $20 \mathrm{~min}$ ensured that very few, if any breaks between bouts were missed. The experiment was repeated using 12 females from another generation.

In the second experiment, the effect of body weight and feeding on sucrose on the duration and time during the night at which they started calling were recorded. Four groups, each consisting of 12 females, were selected of which two consisted of heavy females and two of light females. Female moths emerging on two successive nights were used in this experiment. Those emerging on the first night were weighed and the 12 lightest (hereafter referred to as small; mean weight $=$ $157.63 \pm 2.20 \mathrm{mg}$ ) and heaviest females (hereafter referred to as large; mean weight $=174.41 \pm 1.7 \mathrm{mg}$ ) were selected and singly placed in plastic cylinders as mentioned above. Females of these two groups were given access to water, by placing a 20-ml cup containing a cotton wick immersed in distilled water in each cylinder. On the next night the females that emerged were weighed and the 12 smallest (mean weight $=158.83 \pm 2.28 \mathrm{mg}$ ) and largest females (mean weight $=173.51 \pm 1.24 \mathrm{mg}$ ) were similarly selected and each received a $20-\mathrm{ml}$ cup containing a cotton wick immersed in 15\% (wt : vol) sucrose solution. This procedure yielded four groups of moths: "large, water-fed females" (L-WF), "small, water-fed females" (S-WF), "large, sucrose-fed females" (L-SF) and "small, sucrose-fed females" (S-SF). Calling was recorded over 6 successive nights starting from night 1 . The duration and time of the onset of calling were noted. The cups containing water or sucrose solution were renewed every two days at the beginning of the photophase. This experiment was repeated, with the same sample sizes, using females from another generation. The weights in this replicate were $169.76 \pm 1.31,170.10 \pm 1.35,154.74 \pm 1.91$ and $154.11 \pm 1.73 \mathrm{mg}$ for L-WF, L-SF, S-WF and S-SF, respectively.

\section{Statistical analysis}

Because the results of the two replicates in each experiment were largely consistent, the data were pooled. In the first experiment, the relationships between body weight at emergence and the total time spent calling, and between body weight and longevity, were analyzed using Spearman rank correlation. In the second experiment, ANOVA was used to compare the total time spent calling, time of onset of calling and longevity, of the four groups. Whenever a significant difference was found a Tukey test for multiple comparisons was applied. All calculations were done using Microsoft ${ }^{\circledR}$ Office Excel software and following Sokal \& Rohlf (1981).

\section{RESULTS}

\section{Calling behaviour of starved females in relation to body weight}

None of the starved females called on night 1 and only 3 called on night 6 . However, there was a significant positive correlation between body weight at emergence and total time spent calling on nights 2 to 5 (Fig. 1). Female longevity was also significantly correlated with body weight at emergence (Fig. 2). The statistical values (Spearman correlation coefficient, degrees of freedom and $P$-values) are given on the figures. 


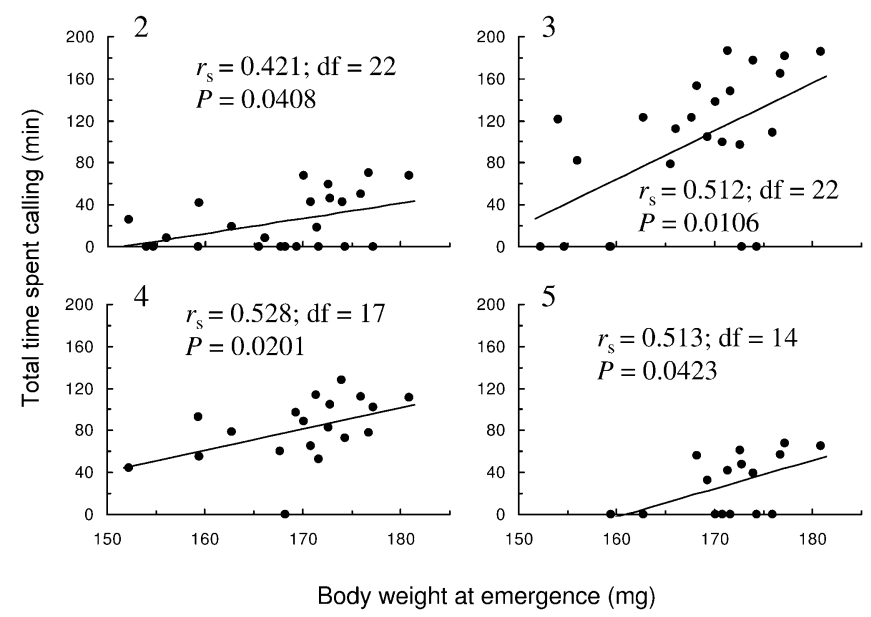

Fig. 1. Correlation between total time females of $S$. littoralis deprived of food or water spent calling and their body weight at emergence. Numbers in the top left hand corners of the figures refer to the number of nights after emergence. On night 1 not all the females called and on night 6 the number of females that called was too few to draw a valid statistical relationship. The Spearman correlation coefficient $\left(r_{\mathrm{s}}\right)$, degrees of freedom (df) and $P$-value are given.

\section{Calling behaviour of sucrose-fed and water fed females in relation to body weight}

The pre-calling period (time from emergence to when they first called) depended on body weight and whether fed or not. The longest pre-calling period was recorded for S-WF and shortest for L-SF (Fig. 3). The four groups of females differed significantly in their pre-calling periods $\left(F_{3,92}=16.07, P<0.0001\right)$. From nights 1 to 4 the time spent calling by L-SF was significantly longer than by L-WF and S-SF, which spent significantly more time calling than S-WF. However, on the nights 5 and 6, the ordering of the total time spent calling was: L-SF $>$ L-WF $>$ S-SF $>$ S-WF (Fig. 4). The time of onset of calling each night generally decreased with age in all groups. On night 2 L-SF started calling significantly earlier than L-WF and S-SF, which started calling significantly earlier than S-WF $\left(F_{3,70}=5.623, P=0.0017\right)$. On night $3 \mathrm{~S}-\mathrm{WF}$ started calling significantly later than the females in the

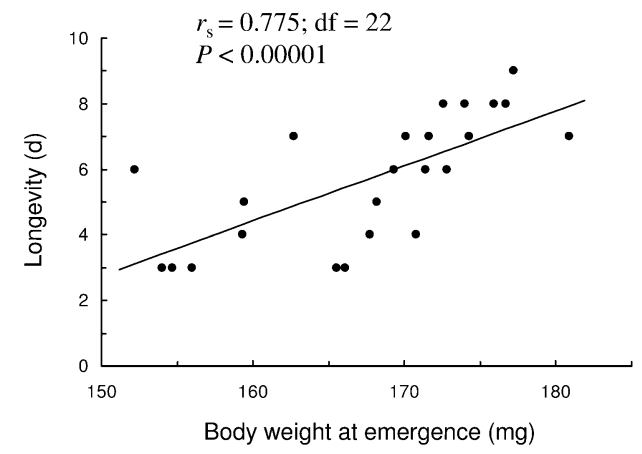

Fig. 2. Correlation between the longevity of females of S. littoralis starved as adults and their body weight at emergence. The Spearman correlation coefficient $\left(r_{\mathrm{s}}\right)$, degrees of freedom (df) and $P$-value are given.

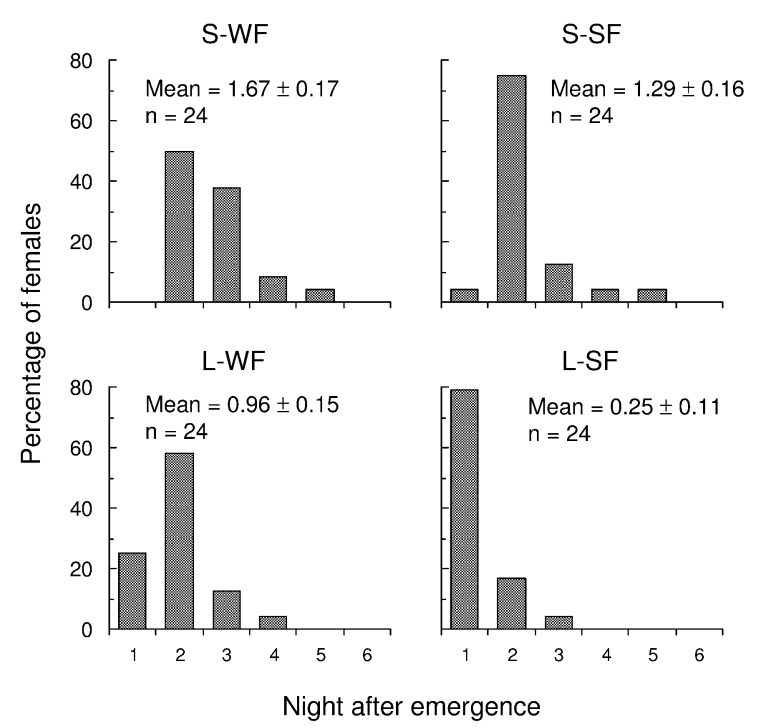

Fig. 3. The temporal distribution of percentage of females calling and the mean length of pre-calling period ( \pm standard error of mean) of females of $S$. littoralis that varied in body weight and whether fed during the adult stage. S-WF is the abbreviation for small water-fed females; S-SF for small sucrose-fed females; L-WF for large water-fed females and L-SF for large sucrose-fed females. The four means are significantly different from each other $\left(F_{3,92}=16.07, P<0.05\right)$.

other three groups $\left(F_{3,86}=5.077, P=0.0028\right)$ (Fig. 5). However, on nights 5 and 6 , the two groups of small females started calling significantly earlier than the two groups of large females $\left(F_{3,89}=6.227, P=0.0007\right.$ and $F_{3,76}=5.532, P=0.0018$, respectively) (Fig. 5). The mean longevity ( \pm standard error) of L-SF, L-WF, S-SF and $\mathrm{S}-\mathrm{WF}$ was $11.51 \pm 0.58,10.17 \pm 0.42,8.12 \pm 0.47$ and $6.88 \pm 0.38 \mathrm{~d}$, respectively. The ANOVA showed that these values are significantly different $\left(F_{3,92}=16.07, P<\right.$ $0.00001)$, and Tukey test revealed that all the values differ from each other $(P<0.05)$.

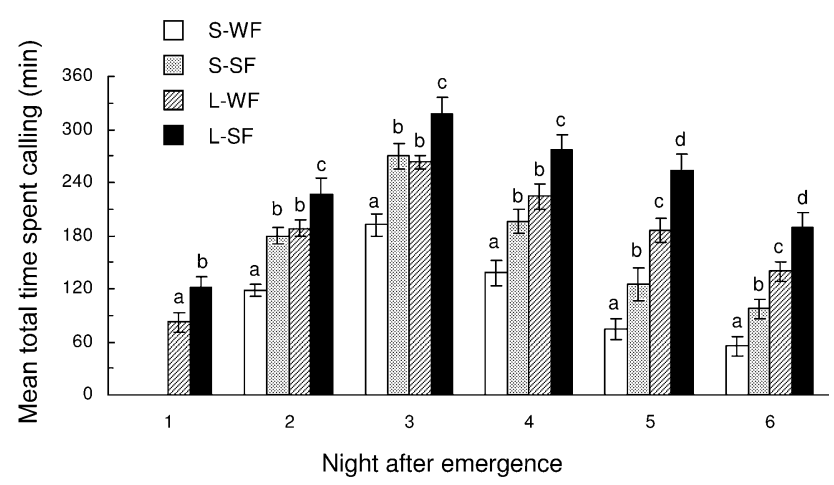

Fig. 4. Effect of feeding as adults and body weight at emergence on the total time spent calling by females of $S$. littoralis recorded over of a period of six nights from the night they emerged. S-WF, S-SF, L-WF and L-SF are the same as in Fig. 3. On night 1 no S-WF called and the number of calling S-SF was too small to derive a valid standard error of mean hence the absence of columns for these females for that night. Columns with different letters are significantly different at $P<0.05$. 


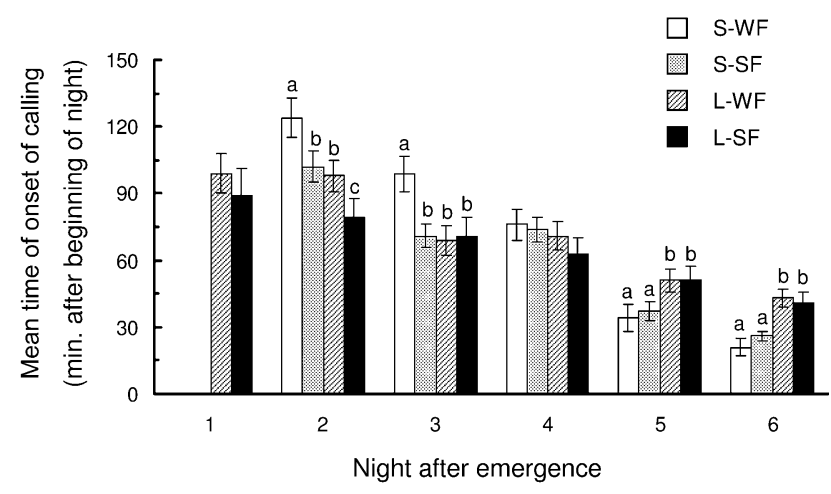

Fig. 5. Effect of feeding as adults and body weight at emergence on the mean time of the onset of calling of females of $S$. littoralis recorded on the six nights following their emergence. S-WF, S-SF, L-WF, and L-SF are the same as in Fig. 3. The absence of columns for S-WF and S-SF on night 1 is due to reasons mentioned in the legend of Fig. 4. Columns with different letters are significantly different at $P<0.05$.

\section{DISCUSSION}

The calling behaviour of female $S$. littoralis was markedly associated with their body weight. There was a strong positive correlation between body weight at emergence and the total time spent calling by starved females on nights 2 to 5 . Large body size is almost universally correlated with high fecundity in insects (Honek, 1993; Tammaru et al., 2002), implying that other aspects of reproductive physiology and behaviour may also be correlated with body weight. Nevertheless, the present study is probably the first to provide empirical evidence that body weight can modulate the calling behaviour of moths. Although the cost of calling is assumed to be low (Cardé \& Baker, 1984; Alberts, 1992) there is indirect evidence that signalling using pheromones is costly. The decrease in pheromone production associated with resistance to pesticides (Delisle \& Vincent, 2002), the use of trehalose and fatty acids as metabolic reservoirs for pheromone biosynthesis (Foster, 2005), the enhancement of sex pheromone production after feeding on sucrose (Foster, 2009; Foster \& Johnson, 2010) and the reduced fecundity and survival associated with calling (Harari et al., 2011) all indicate that calling is metabolically expensive. The long period spent calling by large starved females may thus be due to their having greater metabolic reserves and consequently being more able to sustain long and costly periods of calling.

In addition to the main role of calling as a means of conveying the female's sexual maturity and readiness to mate, pheromones released during calling may also serve as a means of advertising a female's reproductive value. Differences have been recorded in the amounts and ratios of components of the pheromone associated with female body size, age or genetic differences for several species of moth (Löfstedt, 1993; Allison \& Cardé, 2006; Jaffe et al., 2007; Harari et al., 2011). In moths, which are generally nocturnal, it is difficult for males to visually assess at night a female's reproductive value depending on subtle differences in female size, and the female sex pheromone is therefore assumed to be a reliable cue for estimating female quality (Allison \& Cardé, 2008). Large females produce more attractive blends of sex pheromone than small females and males preferentially select and mate with large females (Jaffe et al., 2007; Xu \& Wang, 2009; Harari et al., 2011). Results of the present study further emphasize the significance of body weight in determining mating success, since calling over prolonged periods may enhance the probability of females mating when competing for males (Ambrogi et al., 2009). A positive correlation between body weight and pheromone release is also reported (Huang et al., 1992). In addition, Svensson et al. (1997) have suggested that variation in pheromone production reflects different female strategies. A lowrelease strategy is advantageous when the moths are abundant, but when rare, finding a mate becomes of utmost importance and a high-release strategy may be adopted (Svensson et al., 1997). The fact that female $S$. littoralis possess antennal receptors that detect the species specific sex pheromone (Ljunberg et al., 1993) and the information on the components of the pheromone is processed at the central neuronal level (Sadek et al., 2002), suggest that females may be able to judge population density via their perception of the female-produced pheromone. The experimental design used in the present study included the continuous removal of the sex pheromone produced during calling, which may have mimicked a low population density. Although the release rate, in terms of amount of pheromone emitted per unit time, was not monitored in the present study, lengthy periods of calling may be analogous to high release rates. The ability of large females to call for longer periods than small females implies therefore that large females may be more successful in attracting a mate when the population is sparse.

Both large and small females that were fed sucrose called earlier and for longer than those provided with water. This is rather inconsistent with the reported effects on fecundity of feeding adults sucrose, nectar or honey. Females of the sibling species Spodoptera exempta do not require additional food during the adult stage for maximum egg production, provided the larvae are fed ad libitum (Gunn \& Gatehouse, 1985), which implies that large females (i.e. those well-fed as larvae) do not utilize resources acquired during the adult stage to increase their fecundity. On the other hand, well-fed females of the hawk moth, Amphion floridensis, are more able than poorly-fed females to maximize the use of nectar in enhancing fecundity, because the essential amino acids required for egg production originate entirely from the larval diet (O'Brien et al., 2002). As expected in the present study, however, the increases in the time spent calling by large and small females as a result of ingesting sucrose were both directly proportionate to their size. This may be because calling (the production and release of sex pheromone) does not depend on reserves of nitrogen, like egg production, and as a result calling is less dependent on larval nutritional status than fecundity. The significantly longer time spent calling by sucrose-fed than water-fed females is consistent with the results reported 
by Casimero et al. (2001), which indicate that the calling rate of females fed on honey was significantly greater than of those fed water. The increase in the time spent calling by females fed on sucrose may be a consequence of increased rates of pheromone production. Foster (2009) and Foster \& Johnson (2010) have demonstrated that females fed on sucrose produce more sex pheromone. Regardless of their body weight, moths are expected to utilize sucrose in several ways. Like all animals, moths readily metabolize sugars to acetyl-CoA, an essential compound for the biosynthesis of sex pheromone in Lepidoptera (Tillman et al., 1999). They can also utilize sucrose to derive the energy required for a variety of physiological activities, including the production and release of sex pheromone. Accordingly, even if the longer time spent calling after ingesting sucrose recorded in the present study was a result of increased pheromone production, it remains unclear whether sucrose was metabolized and used as a building block in the production of the pheromone or utilized as a source of energy for the metabolism of stored reserves.

Interestingly, the time spent calling by both sucrose-fed small females and water-fed large females on the first four nights after emergence were comparable. However, ingesting sucrose significantly prolonged the time spent calling by both small and large females on the fifth and sixth nights, but the time spent calling by the small moths was still significantly shorter than by the large moths, regardless of food type. This implies that the ability of large females to increase their calling activity by consuming sucrose-rich foods is less affected by ageing than that of small females. However increasing the likelihood of attracting a mate by calling for longer in early life is more advantageous than attracting a mate later in life. A delay in mating of a few days can greatly reduce a female's reproductive output (Fadamiro \& Baker, 1999; Jones et al., 2008). In S. littoralis a mating delay of a week reduces female fertility to virtually zero (Ellis \& Steele, 1982). Therefore, maximizing the calling activity on the second and third nights may increase the probability of an early mating and hence fitness. Up to the third night after emergence the time when females started calling was also reduced as a result of ingesting sucrose or by being large. This may, at least partly, be due to the relatively longer time the large and/or sucrose-fed females spent calling on these nights. That females start calling earlier in the night as they age is previously reported for this species (Sadek \& Anderson, 2007). This advance in the time of the onset of calling recorded for old females is recorded for several species of moths (McNeil, 1991; Spurgeon et al., 1995; Gemeno \& Haynes, 2000) and is generally viewed as adaptive in that it increases the probability of old females of attracting mates by avoiding competing with young and, supposedly more attractive females (McNeil, 1991 and references therein; Gemeno \& Haynes, 2000). On the fifth and sixth nights the small females started calling significantly earlier than large females, unlike on the earlier nights. There seems to be no obvious reason why the time of onset of calling of the small females decreased with age more progressively than that of large females, irrespective of food type. However, oviposition occurs earlier and the number of eggs laid increases when moths are deprived of food or their wings are clipped, suggesting that reproductive decisions are sensitive to cues of life expectancy (Javoiš \& Tammaru, 2004). From this finding and the results of the present study that small females had significantly shorter lives than large females, as mentioned below, it may be concluded that the tendency of small females to start calling earlier on the fifth and sixth day after emergence is a response to physiological state parameters associated with their relatively short adult life.

For starved females there was a strong positive correlation between body weight and longevity. Large females also lived significantly longer than small females when they were given water or sucrose, and within each weight category sucrose-fed females lived significantly longer than those fed only water. Some studies on other species of moth also record a positive correlation between body weight and longevity (Carroll \& Quiring, 1993; McNamara et al., 2008). One possible reason for the greater longevity of large females is their larger stores of nutrients may help them survive long periods without food. It is also known that the eggs in the ovaries of unmated female moths may be resorbed to increase longevity (Ramaswamy et al., 1997). As the females were not allowed to mate, and large and/or sucrose-fed females are assumed to have relatively large egg loads (Honek, 1993; Tammaru et al., 2002; Xu \& Wang, 2009), it follows that large and/or sucrose-fed females may have lived longer as a result of resorbing relatively large numbers of eggs. Feeding on sucrose is also expected to increase female longevity because it can be used as a fuel. Similarly, both the studies of Fadamiro \& Baker (1999) and Atanassov \& Shearer (2005) indicate that nectar or sugar-fed females live longer than those fed only water.

In summary, the results presented here suggest that large females of $S$. littoralis are probably fitter than small females because they can call for longer, regardless of whether food is available or not. Having access to sucrose also increases the time spent calling and reduces the precalling period. Unlike fecundity, the time spent calling by females increases if they are fed sucrose irrespective of their body weight, implying that even if the food eaten by adults does not directly result in an increase in egg production, it indirectly contributes to the reproductive output. By calling for longer the larger females and those fed sucrose are likely to enhance their likelihood of being mated. The females used in the present study were all virgins and it would be of interest to investigate whether feeding sucrose to small and large mated females would similarly affect the time they spend calling. If so, body weight and feeding during the adult stage should be particularly important in polyandrous species, such as $S$. littoralis (Sadek, 2001), in which a large body and/or feeding on carbohydrate-rich sources may enhance fecundity directly (Leahy \& Andow, 1994; Ramaswamy et al., 1997; Atanassov \& Shearer, 2005), and indirectly by 
increasing longevity and the likelihood of re-mating, which in turn increases fecundity (LaMunyon, 1997; Fadamiro \& Baker, 1999). These results indicate that body weight and adult feeding are important determinants of the ecological and physiological properties of $S$. littoralis and should be considered in future studies on the fitness of moths in natural habitats.

ACKNOWLEDGEMENTS. The author thanks Saber A. Ali for help in the laboratory. The study was partly supported by a SIDA-MENA grant and a Linnaeus Grant obtained via collaboration with the Plant Protection Department, Swedish University of Agricultural Sciences, Alnarp, Sweden.

\section{REFERENCES}

Alberts A.C. 1992: Constraints on the design of chemical communication systems in terrestrial vertebrates. Am. Nat. 139: S62-S89.

AlLISON J.D. \& CARDÉ R.T. 2006: Heritable variation in the sex pheromone of the almond moth, Cadra cautella. J. Chem. Ecol. 32: 621-641.

Allison J.D. \& CARDÉ R.T. 2008: Male pheromone blend preference function measured in choice and no-choice wind tunnel trials with almond moths, Cadra cautella. Anim. Behav. 75: 259-266.

Ambrogi B.G., Fonseca M.G., Coracini M.D.A. \& Zarbin P.H.G. 2009: Calling behaviour and male response towards sex pheromone of poplar moth Condylorrhiza vestigialis (Lepidoptera: Crambidae). J. Pest Sci. 82: 55-60.

Atanassov A. \& Shearer P.W. 2005: Peach extrafloral nectar impacts life span and reproduction of adult Grapholita molesta (Busck) (Lepidoptera: Tortricidae). J. Agric. Urban Entomol. 22: 41-47.

Brown E.S. \& Dewhurst C.F. 1975: The genus Spodoptera (Lepidoptera: Noctuidae) in Africa and the Near East. Bull. Entomol. Res. 65: 221-262.

CARdÉ R.T. \& BAKer T.C. 1984: Sexual communication with pheromones. In Bell W.J. \& Cardé R.T. (eds): Chemical Ecology of Insects. Chapman and Hall, London, pp. 355-383.

CARroll A.L. \& Quiring D.T. 1993: Interactions between size and temperature influence fecundity and longevity of a tortricid moth, Zeiraphera Canadensis. Oecologia 93: 233-241.

Casimero V., NaKasujI F. \& FujISAKI K. 2001: The influences of larval and adult food quality on the calling rate and precalling period of females of the cotton bollworm, Helicoverpa armigera Hübner (Lepidoptera: Noctuidae). Appl. Entomol. Zool. 36: 33-40.

Delisle J. \& Vincent C. 2002: Modified pheromone communication associated with insecticidal resistance in the obliquebanded leafroller, Choristoneura rosaceana (Lepidoptera: Tortricidae). Chemoecology 12: 47-51.

Ellis P.E. \& Steele G. 1982: The effects of delayed mating on fecundity of females of Spodoptera littoralis (Boisduval) (Lepidoptera: Noctuidae). Bull. Entomol. Res. 72: 295-302.

FADAMIRO H.Y. \& BAKER T.C. 1999: Reproductive performance and longevity of female European corn borer, Ostrinia nubilalis: Effects of multiple mating, delay in mating, and adult feeding. J. Insect Physiol. 45: 385-392.

FosTer S.P. 2005: Lipid analysis of the sex pheromone gland of the moth Heliothis virescens. Arch. Insect Biochem. Physiol. 59: $80-90$.

Foster S.P. 2009: Sugar feeding via trehalose haemolymph concentration affects sex pheromone production in mated Heliothis virescens moths. J. Exp. Biol. 212: 2789-2794.
Foster S.P. \& Johnson C.P. 2010: Feeding and hemolymph trehalose concentration influence sex pheromone production in virgin Heliothis virescens moths. J. Insect Physiol. 56: 1617-1623.

Gemeno C. \& Haynes K.F. 2000: Periodical and age related variation in chemical communication system of black cutworm moth, Agrotis ipsilon. J. Chem. Ecol. 26: 329-342.

Gómez V.R.C. \& RoJAs J.C. 2006: Calling behavior of Zamagiria dixolophella (Lepidoptera: Pyralidae). Florida Entomol. 89: 83-84.

Gu H. \& Danthanarayana W. 1990: The role of availability of food and water to the adult Epiphyas postvittana, the light brown apple moth, in its reproductive performance. Entomol. Exp. App. 54: 101-108.

GunN A. \& Gatehouse A.G. 1985: Effects of the availability of food and water on reproduction in the African armyworm, Spodoptera exempta. Physiol. Entomol. 10: 53-63.

HARARI A.R., ZAhAVI T. \& ThiÉRY D. 2011: Fitness cost of pheromone production in signaling female moths. Evolution 65: $1572-1582$.

HoneK A. 1993: Intraspecific variation in body size and fecundity in insects: a general relationship. Oikos 66: 483-492.

Huang C.B., Li W.G. \& Fu W.J. 1992: The release rate and component ratio of sex pheromone in pink bollworm of different seasonal generations. Acta Entomol. Sin. 35: 279-284.

Jaffe K., Mirás B. \& Cabrera A. 2007: Mate selection in the moth Neoleucinodes elegantalis: evidence for a supernormal chemical stimulus in sexual attraction. Anim. Behav. 73: 727-734.

JAVOIŠ J. \& TAMmARU T. 2004: Reproductive decisions are sensitive to cues of life expectancy: the case of a moth. Anim. Behav. 68: 249-255.

Jones V.P., Wiman N.G. \& BRUNNer J.F. 2008: Comparison of delayed female mating on reproductive biology of codling moth and oblique banded leafroller. Environ. Entomol. 37: 679-685.

LAMunYon C.W. 1997: Increased fecundity, as a function of multiple mating, in an arctiid moth, Utetheisa ornatrix. Ecol. Entomol. 22: 69-73.

LEAHY T.C. \& ANDOW D.A. 1994: Egg weight, fecundity, and longevity are increased by adult feeding in Ostrinia nubilalis (Lepidoptera: Pyralidae). Ann. Entomol. Soc. Am. 87: 342-349.

Lim H., Park K.C., Baker T.C. \& Greenfield M.D. 2007: Perception of conspecific female pheromone stimulates female calling in an arctiid moth, Utetheisa ornatrix. J. Chem. Ecol. 33: $1257-1271$.

Liu Z.D., Li D.M., Gong P.Y. \& Wu K.J. 2004: Life table studies of the cotton bollworm, Helicoverpa armigera (Hübner) (Lepidoptera: Noctuidae), on different host plants. Environ. Entomol. 33: 1570-1576.

Ljungberg H., Anderson P. \& Hansson B.S. 1993: Physiology and morphology of pheromone-specific sensilla on the antennae of male and female Spodoptera littoralis (Lepidoptera: Noctuidae). J. Insect Physiol. 39: 253-260.

LÖFSTEDT C. 1993: Moth pheromone genetics and evolution. Phil. Trans. R. Soc. Lond. (B) 340: 167-177.

McNamara K.B., Elgar M.A. \& Jones T.M. 2008: Seminal compounds, female receptivity and fitness in the almond moth, Cadra cautella. Anim. Behav. 76: 771-777.

McNamara K.B., Elgar M.A. \& Jones T.M. 2009: Large spermatophores reduce female receptivity and increase male paternity success in the almond moth, Cadra cautella. Anim. Behav. 77: 931-936. 
McNeIL J.N. 1991: Behavioral ecology of pheromone-mediated communication in moths and its importance in the use of pheromone traps. Ann. Rev. Entomol. 36: 407-430.

Mozuraitis R. \& Buda V. 2006: Pheromone release behaviour in females of Phyllonorycter junoniella (Z.) (Lepidoptera, Gracillariidae) under constant and cycling temperatures. $J$. Insect Behav. 19: 129-142.

O'brien D.M., Schrag D.P. \& Rio C.M.D. 2000: Allocation to reproduction in a hawkmoth: a quantitative analysis using stable carbon isotopes. Ecology 81: 2822-2831.

O’Brien D.M., Fogel M.L. \& Boggs C.L. 2002: Renewable and nonrenewable resources: Amino acid turnover and allocation to reproduction in Lepidoptera. Proc. Natl. Acad. Sci. USA 99: 4413-4418.

Ramaswamy S.B., Shu S., Park Y.I. \& Zeng F. 1997: Dynamics of juvenile hormone-mediated gonadotropism in the Lepidoptera. Arch. Insect Biochem. Physiol. 35: 539-558.

REAVEY D. 1993: Why body size matters to caterpillars. In Stamp N.E. \& Casey T.M. (eds): Caterpillars - Ecological and Evolutionary Constraints on Foraging. Chapman and Hall, New York, pp. 249-279.

Ryne C., Nilsson P.A. \& Siva-Jothy M.T. 2004: Dietary glycerol and adult access to water: effects on fecundity and longevity in the almond moth. J. Insect Physiol. 50: 429-434.

SADEK M.M. 2001: Polyandry in field-collected Spodoptera littoralis moths and laboratory assessment of the effects of male mating history. Entomol. Exp. Appl. 98: 165-172.

SADEK M.M. 2011: Complementary behaviors of maternal and offspring Spodoptera littoralis: Oviposition site selection and larval movement together maximize performance. $J$. Insect Behav. 24: 67-82.

SAdek M.M. \& Anderson P. 2007: Modulation of reproductive behavior of Spodoptera littoralis by host and non-host plant leaves. Basic Appl. Ecol. 8: 444-452.

SadeK M.M., Hansson B.S., Rospars J.-P. \& Anton S. 2002 Glomerular representation of plant volatiles and sex pheromone components in the antennal lobe of the female Spodoptera littoralis. J. Exp. Biol. 205: 1363-1376.
Salama H.S. \& Shoukry A. 1972: Flight range of the moth of the cotton leaf worm Spodoptera littoralis (Bois). Z. Angew. Entomol. 71: 181-184.

SoKal R.R. \& Rohlf F.J. 1981: Biometry. The Principles and Practique of Statistics in Biological Research. 2nd edn. W.H. Freeman, New York, 859 pp.

Spurgeon D.W., Lingren P.D., Raulston J.R. \& Shaver T.N. 1995: Age-specific mating activities of Mixican rice borers (Lepidoptera: Pyralidae). Environ. Entomol. 24: 105-109.

Svensson M.G.E., Bengtsson M. \& LöfQvist J. 1997: Individual variation and repeatability of sex pheromone emission of female turnip moth Agrotis segetum. J. Chem. Ecol. 23: 1833-1850.

Tammaru T., Esperk T. \& Castellanos I. 2002: No evidence for costs of being large in females of Orgyia spp. (Lepidoptera, Lymantriidae): larger is always better. Oecologia 133: 430-438.

Teng Z.Q. \& Zhang Q.W. 2009: Determinants of male ejaculate investment in the cotton bollworm Helicoverpa armigera: mating history, female body size and male age. Physiol. Entomol. 34: 338-344.

Thiery D. \& Moreau J. 2005: Relative performance of European grapevine moth (Lobesia botrana) on grapes and other hosts. Oecologia 143: 548-557.

Tillman J.A., Seybold S.J., Jurenka R.A. \& Blomquist G.J. 1999: Insect pheromones - an overview of biosynthesis and endocrine regulation. Insect Biochem. Mol. Biol. 29: 481-514.

Torres-Vila L.M., Stockel J. \& Rodríguez-Molina M.C. 1997: Physiological factors regulating polyandry in Lobesia botrana (Lepidoptera: Tortricidae). Physiol. Entomol. 22: 387-393.

Torres-Vila L.M., Rodríguez-Molina M.C., McMinn M. \& RodRíGueZ-Molina A. 2005: Larval food source promotes cyclic seasonal variation in polyandry in the moth Lobesia botrana. Behav. Ecol. 16: 114-122.

WheELER D. 1996: The role of nourishment in oogenesis. Ann. Rev. Entomol. 41: 407-431.

XU J. \& WANG Q. 2009: Male moths undertake both pre- and incopulation mate choice based on female age and weight. Behav. Ecol. Sociobiol. 63: 801-808.

Received August 19, 2011; revised and accepted October 4, 2011 